\title{
Directing transcription to the right way
}

\author{
Cell Research (2013) 23:1153-1154. doi:10.1038/cr.2013.100; published online 30 July 2013
}

How cells ensure that productive transcription from divergent promoters is limited to the downstream protein-coding region is an important question in the transcription field. A recent study in Nature proposed an answer by revealing that the upstream antisense transcripts undergo early termination through the polyadenylation signal-dependent pathway, and the downstream sense transcripts are protected from premature cleavage by $\mathrm{U} 1$ small nuclear ribonucleoprotein (snRNP).

It is common in mammals and yeast that transcription initiates in two opposite directions from the same promoter, which is defined as a divergent promoter. This phenomenon is proposed to play a role in transcriptional regulation by maintaining the nucleosome free regions (NFR) and producing negative supercoils within promoters (reviewed in [1]). In metazoans, RNA polymerase II (RNAPII) is paused in close proximity to the +1 nucleosome at the border of NFR after transcription is initiated and RNAPII is phosphorylated at Ser5 on its C-terminal domain (CTD) by Cdk7. The paused RNAPII has been shown to compete with nucleosomes for occupancy at promoters, as the promoter regions are less accessible to MNase digestion when the paused RNAPIIs are lacking [2]. Therefore, divergent transcription, with polymerases pausing at the upstream -1 and the downstream +1 nucleosomes, may reinforce the boundaries of NFR and facilitate further rounds of initiation. In addition, negative supercoils will form behind an elongating polymerase, and the negative superhelical tension can assist DNA unwinding in the promoter, facilitating transcription initiation [3]. By transcribing both the upstream and downstream regions in opposite directions, divergent transcription may build up sufficient negative supercoils within the promoter to separate the DNA strands, and therefore increase the transcription efficiency.

Although divergent transcription occurs from most active promoters, the abundance of the upstream antisense RNAs (uaRNAs) is much lower compared to the downstream protein-coding mRNAs [4-6]. Both transcriptional and post-transcriptional mechanisms contribute to the downregulation of uaRNA expression. Preker et al. [5] demonstrated that uaRNAs are constantly degraded by exosome, as depletion of an exosome subunit hRrp40 led to the stabilization of uaRNAs. On the other hand, although P-TEFb, a kinase phosphorylating Ser2 of the RNAPII CTD and releasing RNAPII from the pause site, is recruited to both the upstream and the downstream RNAPIIs at divergent promoters [7], a global runon sequencing (GRO-seq) analysis did not detect upstream transcription going beyond $1500 \mathrm{bp}$ from the transcription start sites (TSSs) of the protein-coding genes [4]. Also, while H3K4 trimethylation (H3K4me3), a histone mark for initiation, is associated with the paused RNAPIIs on both sides of the active promoters , localization of the elongation mark H3K79me2 is limited to the downstream coding regions [6]. This evidence indicates that the divergent transcription may undergo successful initiation and early elongation, but the productive elongation only occurs downstream over the genes. In yeast, DNA looping has been shown to play a role in the selective transcriptional directionality. Tan-Wong et al. [8] demonstrated that loss of gene loops by mutating either individual genes or Ssu72, which associates with both the promoter and the terminator and is required for gene looping, resulted in increased expression levels of uaRNAs. However, whether this mechanism is conserved in higher organisms or other alternative mechanisms are utilized still remains unclear.

Recently, Almada et al. [9] revealed that the early termination of upstream antisense transcription contributes to the directionality of divergent promoters in mouse embryonic stem cells. Deep sequencing of the $3^{\prime}$ ends of polyadenylated RNAs was used to examine the transcript cleavage sites close to the TSSs, and an interesting phenomenon was uncovered: the upstream antisense regions have 2 -fold more cleavage sites as compared to the downstream sense regions. The number of upstream cleavage sites peaks at about 700 bases from the TSS of the downstream gene, suggesting that the uaRNAs undergo termination in the early stage of elongation. This early termination of uaRNAs is mediated through the canonical poly(A) site (PAS)-dependent pathway, as the distribution of the cleavage and polyadenylation machinery on chromatin are very similar to the positional profile of uaRNA cleavage sites, and the upstream antisense regions show a 33\% enrichment of the PAS sites as compared to the coding regions. In addition, discriminative hexamer analysis revealed that the sense genes are enriched with $5^{\prime}$-splice- 


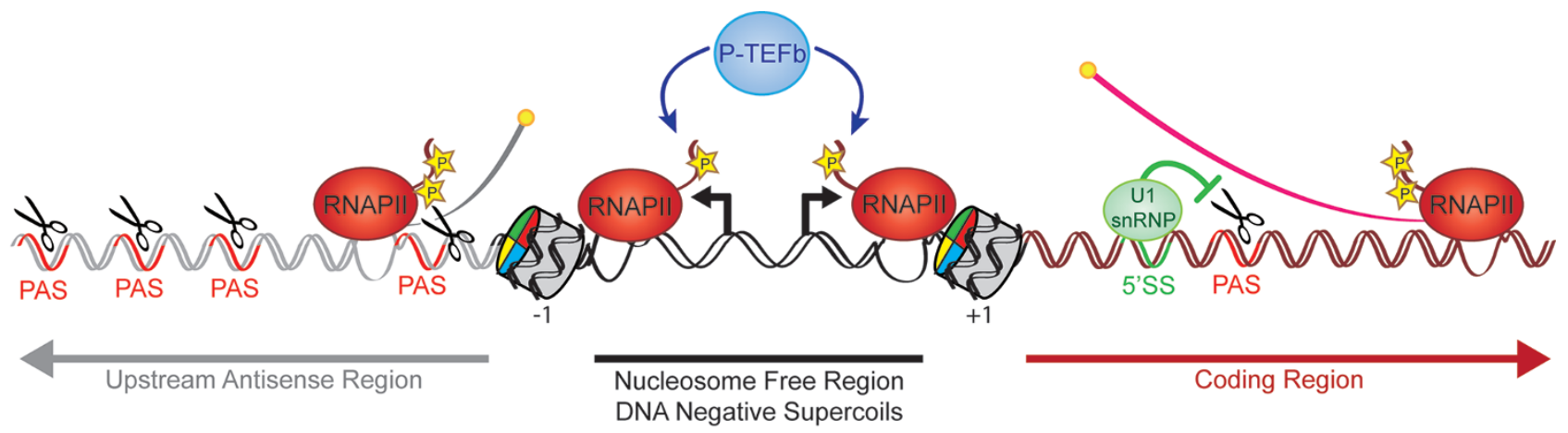

Figure 1 RNAPIIs are initiated in two opposite orientations from an active divergent promoter, and paused at the -1 and +1 nucleosomes, respectively. The paused RNAPIIs reinforce the boundaries of NFR and maintain the accessibility of promoter to transcription factors. P-TEFb is recruited to both upstream and downstream paused RNAPIls and releases them from the pause sites. The elongation by RNAPIIs produces negative supercoils in the promoter region, facilitating further rounds of initiation. Shortly after released from the pause site, the upstream RNAPII transcribes through the PAS, and the transcription is terminated by the canonical 3'-endprocessing factors (Left; upstream antisense region). In the downstream coding region (Right), the PAS is suppressed by the U1 snRNP recruited by 5 'SS, and therefore productive elongation occurs through the protein-coding gene.

site (5'SS)-related sequences, which are recognized by U1 snRNP. Interestingly, other than functioning in RNA splicing, U1 snRNP has been reported to suppress premature termination of pre-mRNA by the canonical cleavage and polyadenylation machinery [10]. This protective property of U1 snRNP is crucial for regulating the promoter directionality, as blocking the U1 snRNP-5'SS interaction resulted in a dramatic increase in the cleavage sites on the downstream sense strand, eliminating the differences in the cleavage events between uaRNAs and mRNAs. Moreover, the depletion of PAS and the enrichment of 5'SS in the protein-coding regions can explain the asymmetry of the cleavage events between the two sides of promoters by simulation, indicating that the U1-PAS axis is an important mechanism that governs the directionality of divergent promoters.

Divergent transcription may promote active transcription by facilitating initiation, and its "side effects", the potentially resource-wasting uaRNAs, are eliminated by early termination followed by exosome-mediated degradation (Figure 1), providing an elegant example of how biological mechanisms are integrated to benefit from a process but also prevent damage from it. Nevertheless, more questions await answers, as other mechanisms such as gene looping may also contribute to the regulation of promoter directionality in mammals. Given the protection range of $\mathrm{U} 1 \mathrm{snRNP}$ predicted by simulation to be as large as $1 \mathrm{~kb}$, its suppressive effects on transcriptional termination may be indirect or the recognition of 5'SS by U1 snRNP may elicit local changes in chromatin conformation. Taken together, further advances in the knowledge of divergent transcription will provide a broader and more complete view of transcriptional regulation.
Fu Huang ${ }^{1}$, Jerry L Workman ${ }^{1}$

${ }^{1}$ Stowers Institute for Medical Research, 1000 East 50th Street, Kansas City, MO 64110, USA

Correspondence: Jerry L Workman

E-mail: jlw@stowers.org

\section{References}

1 Seila AC, Core LJ, Lis JT, et al. Cell Cycle 2009; 8:2557-2564.

2 Gilchrist DA, Dos Santos G, Fargo DC, et al. Cell 2010; 143:540-551.

3 Parvin JD, Sharp PA. Cell 1993; 73:533540.

4 Core LJ, Waterfall JJ, Lis JT. Science 2008; 322:1845-1848.

5 Preker P, Nielsen J, Kammler S, et al. Science 2008; 322:1851-1854.

6 Seila AC, Calabrese JM, Levine SS, et al. Science 2008; 322:1849-1851.

7 Preker P, Almvig K, Christensen MS, et al. Nucleic Acids Res 2011; 39:7179-7193.

8 Tan-Wong SM, Zaugg JB, Camblong J, et al. Science 2012; 338:671-675.

9 Almada AE, Wu X, Kriz AJ, et al. Nature 2013; 499:360-363.

10 Kaida D, Berg MG, Younis I, et al. Nature 2010; 468:664-668. 